\title{
Visualization of the Phase Volume Distribution in Alloys
}

\author{
Makarenko K.V., Kuzovov S.S., Nikitin A.A. \\ makkon1@yandex.ru| kss41188@inbox.ru|zzzalexzzz95@gmail.com \\ BSTU, Bryansk, Russian Federation
}

\begin{abstract}
The method of obtaining the volume distribution of phases in structural materials is considered. The technique is based on the layerby-layer grinding of metallographic samples with photo fixation of microstructure images and the subsequent computer processing of these images. An example of using the developed methodology to study the features of the volumetric distribution of graphite inclusions in high-strength cast irons is presented. The classification of graphite inclusions depending on the shape, size and distribution features is given. The study of the features of the distribution of graphite inclusions led to the conclusion about the patterns and mechanisms of formation graphite phase in cast iron.
\end{abstract}

Keywords: visualization, tomography, volume distribution, phase, materials, cast iron, graphite.

\section{Introduction}

The methods of obtaining the idea about the volume distribution of phases in different structural materials have undergone considerable evolutionary development in recent decades. Basic principles and approaches were laid down in the works by S.A.Saltykov [9]. The methods which were used by stereometric metallography were characterized by high labor intensity and required from the researcher special preparation and thorough execution of the developed methods. However, the results which were obtained in the course of present investigation, widened considerably the idea (conception) about the processes going in the materials, and allowed to maintain important relations between their structure and properties. For example, the works by K.P.Bunin with his pupils [1], allowed to get the idea about morphology of graphite inclusions in grey cast irons.

The wide introduction of computer methods of processing the images simplified considerably the methods and visualizations of phase volume distribution in alloys. However, the approaches remained the same.

At the present time an individual direction named as a three-dimensional one $[5,12,6]$ is developing intensely in the material science. As follows from the name, with such an approach the structures of the described materials have a 3dimensional (volume) image. Undoubtedly, such an approach allows to obtain a more integral and realistic idea about the structure of the materials and processes going in them $[11,3,4,2,8]$

The main method which is used by the new direction in the material science is a computer computational tomography [7]. Tomography used in the material science, uses different physical processes and methods for investigation of internal structure of materials: X-ray, magnetic-resonance, acoustic ones and a traditional one based on the mechanical layered grinding the material with obtaining 2-dimensional images of the structure and their subsequent summation and transition to the volume representation [9].

In the present article the methods is presented based on the latter approach of computer tomography. The developed methods was used for the study of peculiarities of distribution of the graphite phase in high-strength cast irons.

\section{Methods of Holding the Investigation}

For the study of volume distribution of graphite phase in cast irons the cast iron was made in the production conditions of the cast iron workshop of the CJSC «UK BMZ». The specimens were investigated from the cast iron which was produced by means of induction melting in the furnace PVK-NK, based on the remelting of conversion pig iron PVK-NK and steel C245. As a carburizer, the graphitized coke breeze was used. Inoculation was made in the ladle by the sandwich process, the inoculant FeSiMg-7. The average chemical composition of the investigated cast iron, \% mass: $3.17 \mathrm{C} ; 3.3 \mathrm{~S} ; 0.76 \mathrm{Mn}, 0.06 \mathrm{P}$; $0.03 \mathrm{~S} ; 0.05 \mathrm{Mg}$.

Cast iron was cast into sand and loam moulds for making the castings of different shapes and blanks in the form of the block of size 500x500x1000. From the blanks were obtained the specimens for metallographic investigations, in the castings were investigated the microstructure of special boss specimens and elements of pouring gate systems.

Subsequent investigations of the volume distribution of graphite inclusions were carried out on one specimen cut out from the blank, with the use of methods of optical tomography (fig.1)

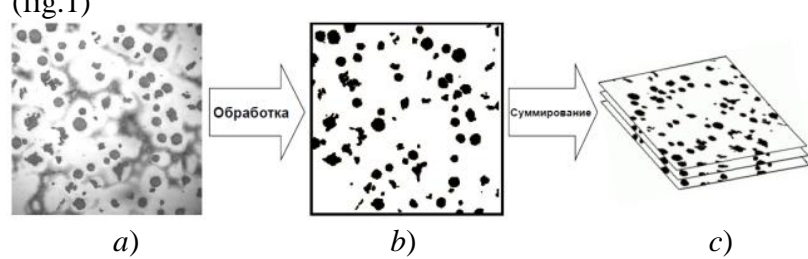

Fig.1. Stages of processing the images of the microstructure of the high-strength cast iron:

a) - initial image of microstructure $\mathrm{x} 80$;

b) - transformation to binary image of graphite inclusions distribution in the plane of the metallographic specimen;

c)- summation of binary images

As reference points served the imprints of introduction of the hardness tester indenter TK-2M in the surface layers of the metallographic specimen. For obtaining the idea about volume location of the graphite phase the layered machining was made on the grinding-and-polishing machine-tool TIME PG-1000. The calculation of the depth of grinding was determined by the change of the diameter of the harness tester indenter imprint (fig.2). The diameter of the imprint was determined by means of the special eyepiece with the scale, for the graduation of which a reference object (micrometer) was used.

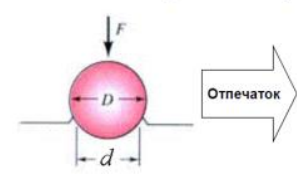

a)

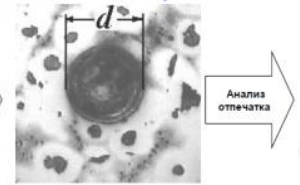

b)

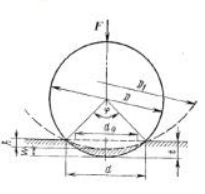

c)
Fig. 2. Methods of determination of the grinding depth:

a) - diagram of introduction of hardness tester indenter and obtaining the imprint;

b) - photo of initial imprint of indenter on the surface of the investigated specimen;

c) - geometric diagram of determination of depth at successive grinding of the specimen along the change of indenter imprint diameter. 
Distribution of graphite was studied on the non-etched metallographic specimen with the help of the inverted metallographic microscope Nikon ECLIPSE MA 200 with magnification $\mathrm{x} 80$. The observed microstructure was fixed with the help of the digital camera built into the microscope. The next processing of the obtained photos was made with the help of program Above Photoshop and Image J. For obtaining the image of volume distribution of graphite inclusions in the matrix of the cast iron was used the program module Image $\mathbf{J}$, for rendering the volume images - the module Volume J.

\section{Results of Investigations}

The results of computer processing of the microstructure are presented in fig.3. Unfortunately, such an image does not allow to evaluate fully the picture of volume distribution of the graphite phase in cast irons, because graphite inclusions on different levels obstruct each other

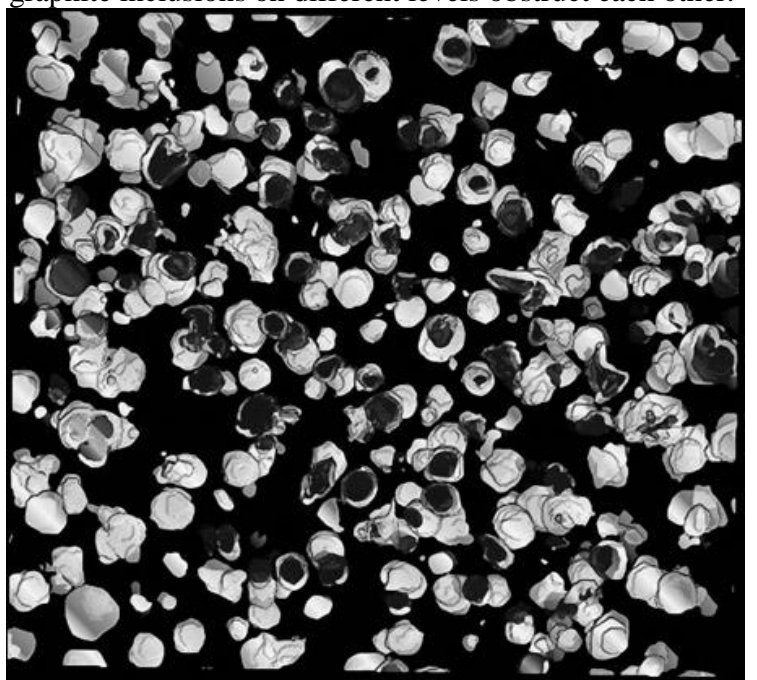

Fig.3. Volume distribution of graphite inclusions in highstrength cast iron with spheroidal graphite

The next analysis was carried out on separate levels with division of initial mass into 2 separate layers. The results of the analysis in the form of projections of isolines of graphite inclusions are presented in fig. $4, a$ and $b$.

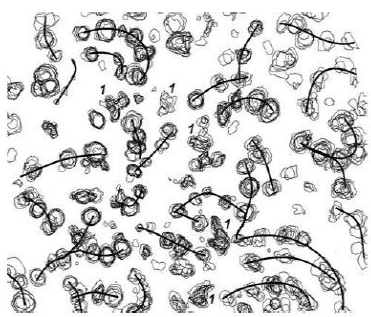

a)

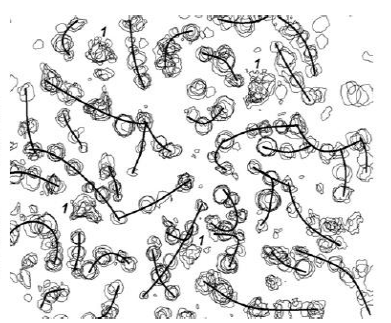

b)
Fig.4. Layered distribution of graphite inclusions in highstrength cast iron:

a) - layer consists of 10 images;

b) - layer consists of 8 images

\section{Discussion of Results}

During the analysis of images (fig.4) the fact draws attention that the chains of inclusions are represented by graphite spheroids of approximately the same size. Apparently, such graphite inclusions making the chain, were nucleated simultaneously and crystallized in the same conditions. In the cast iron structure the chains occur which are represented only by small inclusions, or small inclusions are at the beginning (at the end) of the chain. It is explained by the fact that on the metallographic specimens at the layered grinding the complete inclusion is not present wholly, but only the projections of the cut (section) corresponding to the upper or lower part of the graphite spheroid, cut by the plane of the metallographic specimen during investigation. The observed chains do not have any generalizing regularities in structure. By topological structure they form nuclear complexes passing into each other which bifurcate and inosculate, or grow (swell) in layers. Under the cell in this case the eutectic cell is meant.

Besides the spheroidal graphite, in the cast iron microstructure are present inclusions of irregular compact shape. They have comparatively big sizes. It means that in the process of growth they do not lack in carbon. Such graphite, as a rule, is not included into chains and is located in the microstructure apart (fig.4, $a$ and $b$ ).

Location and shape of graphite inclusions of nonspheroidal shape show that they appear at the final stage of hardening the cast iron, in the conditions not allowing their spheroidizing because of the lack of $\mathrm{Mg}$.

Besides the above-mentioned graphite forms, in the cast iron microstructure the areas occur with especially big inclusions, fig. 4 , $a$ and $b$. The prevailing size shows that graphite spheroids are initial. The composition of the investigated cast iron is eutectic one, however, the elements of structure of hypereutectic cast iron are present. Formation of excessive, even for layers of eutectic composition, primary phases was also observed earlier, in particular, in systems $\mathrm{Al}-\mathrm{Si}, \mathrm{Pb}-\mathrm{Bi}$ and $\mathrm{Zn}-\mathrm{Gd}$ [10]. The arisen contradiction is explained by a considerable deviation in the processes of hardening of real production alloys from balanced conditions of crystallization of eutectic alloys. The main reason which results in appearing the primary crystals of phases is fluctuation of chemical composition. For cast irons the increase of stability of chemical composition fluctuations is conditioned by additional inoculation. Initially in the melt $\mathrm{Fe}-\mathrm{C}$-Si there are areas with different content of carbon and silicon, hereby the areas enriched with silicon are depleted with carbon, and vice versa; this is connected with influence of silicon on thermodynamic activity of carbon. At inoculation with silicon-containing spheroidizating alloying compositions to which also refers the inoculant FeSiMg7 , the process of heterogenisation increases. In the areas with increased concentration of carbon graphite inclusions start forming, as the concentration of carbon in these areas exceed ultimate solubility in the iron melt at this temperature. Fluctuations of the content result in arising not only individual inclusions of primary graphite (fig. $4, a$ ), but also in formation of spheroidal cluster consisting of several primary inclusions (fig.4, $b$ ). The structure of the cluster presented by the chains of graphite inclusions, shows the unified mechanism of distribution of inclusions of primary and eutectic graphite.

Thus, graphite inclusions observed in the cast iron microstructure can be classified by the following kinds: I - cluster and individual inclusions of primary graphite of spheroidal shape; II - spheroidal inclusions of eutectic graphite and III -large compact inclusions of non-spheroidal shape.

Based on classification of graphite inclusions, the pattern of distribution of graphite phase in the high-strength cast iron is developed. Morphological peculiarities of the structure of graphite inclusions, observed on metallographic specimens, can be explained by the fact that graphite in the cast iron forms in several stages.

At the initial stage of crystallization after inoculation occurs heterogenization of the melt by silicon and carbon. Heterogenization of the melt results in its separation into layers. In the areas with increased concentration of carbon the centres of crystallization of graphite start to arise. The subsequent 
overcooling, which arises at the inoculation of the melt with magnesium-containing alloying compositions, stabilize the nuclei of graphite inclusions. The nuclei of graphite phase grow with big speeds on account of accelerated diffusion of carbon and get big sizes. Graphite inclusions arising in the melt volume, limited by fluctuation, during the contact which is caused by their growth and the size of fluctuation, form spherical cluster at the moment preceding formation of the austenite shells. In case when sizes of arising fluctuation are comparatively small, a single inclusion of primary graphite forms in it. Hereby, in case of formation of several centres of crystallization of graphite in a small area of the melt, occurs their accretion into one big inclusion in accordance with the mechanism of overcondensation.

At the next stage in the areas of the melt with chemical composition corresponding to eutectic content of the alloy components, eutectic cells start to form, which present by themselves graphite of spheroidal shape covered with shells of crystallizing austenite. Graphite nuclei in the melt areas arise evenly, the process of coalescence, which aligns average sizes of centres of graphite crystallization, prevents them from segregation.

In case of arising, beside the growing graphite nucleus, of another smaller one, a gradual enlargement of the smaller one is observed, on account of diffusion of carbon from small nuclei to big ones, which arose earlier. Graphite inclusions form at the big rates of overcooling and are covered with austenite shell earlier than primary graphite, which slows down their subsequent growth. Chemical composition of austenite surrounding graphite inclusions, has the increased concentration of silicon, which confirms the fact of formation of ferrite areas around graphite, the so-called " a bull's eye "structure. The growth and formation of graphite inclusions at this stage occurs in the areas of the melt with the increased content of silicon.

The organization of the chains of eutectic graphite, observed on the metallographic specimen is caused by weldability of austenite shells and their subsequent joint growth during crystallization. At this stage, due to the shortfall of carbon duffusing through austenite shells, the neighbouring (competitive) graphite inclusions can get the shape of ellipsoids and form the necks uniting them (fig.5).

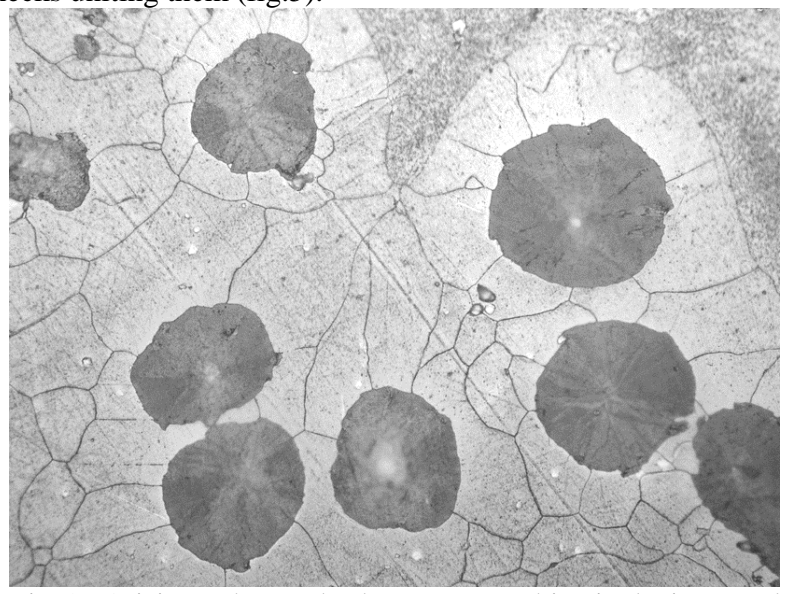

Fig.5 Arising the necks between graphite inclusions and transformation of spheroids into ellipsoids (heat etching, $\mathrm{x} 500)$

Mutual weldability of the neighbouring austenite shells of eutectic nuclei happens due to the striving of the system to decreasing the inter-phase energy. Graphite inclusions arisen in the melt, accrete with austenite shells and join the existing cluster chains. The chains of graphite inclusions observed on the surface of the metallographic specimen, arise in the result of gradual welding to them of new eutectic nuclei, which are formed in the areas of the melt, limited by increased concentration of silicon.
The structures of chains of graphite inclusions, formed in the process of crystallization of the high-strength cast iron, are, by themselves, fractal clusters, which can be described with the help of the CCA pattern (Cluster-Cluster Aggregation). In this pattern the particles colliding with each other, form originally simple clusters, which later unite in the clusters of big sizes. The clusters arising in the melt, consisting in eutectic nuclei, manifest themselves most clearly in the flaws of shrink origin. In shrink cavities, at the condition when the mother liquor, enriched with segregated materials and carbon, is removed in the process of shrinkage, the crystallized welded cluster chains of eutectic grains are found in the shrink porosity. This is the proof of the suggested cluster pattern of distribution of graphite inclusions in highstrength iron.

Big graphite of irregular compact shape is crystallized at the last stage from the mother melt, in the areas stuck by growing austenite-and-graphite eutectic aggregates. The proof of crystallization of graphite from the mother liquor are the fringes of graphite observed in shrink cavities. Graphite crystallizing at the last stage, is surrounded, as a rule, by the pearlite structure and does not have any ferrite fringes of the "bull's eye" type. The conditions of formation of graphite inclusions do not contribute to spheroidization of graphite, and it degenerates into the compact, close to vermicular, X-shape. Apparently, the last portions of metal crystallize without concentration of residual magnesium, required for spheroidization of graphite.

\section{Conclusion}

Methods of visualization of phase volume distribution in alloys is developed. The suggested method is used for the analysis of peculiarities of graphite phase distribution in highstrength cast irons and for the study of crystallization process. The processes of primary structure formation of graphite phase in high-strength cast irons are investigated. At the use of methods of stereology and digital metallography was obtained 3D-distribution of graphite inclusions in the cast iron. The classification of graphite inclusions dependent on the shape, sizes and peculiarities of their distribution, is stated. The hypothesis is proposed about the fact that structures of chains of graphite inclusions forming in the process of crystallization of the high-strength cast iron, are actually fractal clusters which can be described with the help of the CCA pattern (ClusterCluster Aggregation).

\section{List of References}

[1] Bunin K.P., Malinochka Ya.N., Taran Yu.N. (1969) Osnovi metallografii chuguna [The Basics of Cast Iron Metallography] - M., Metallurgy, -415 p.

[2] Cerqueira, F.A. Three-dimensional reconstruction of compacted graphite in vermicular cast iron by manual serial sectioning/Fernando Almeida Cerqueira, Arthur Seiji Nishikawa, Wilson Luiz Guesser, Cesar Roberto de Farias Azevedo//Metallurgy and Materials, 2015№3 - p.307-312.

[3] Domitner,J. 3D Simulation of interdendritic flow through a Al.-18 wt\% Cu structure captured with X-ray microtomography/ J.Domitner, C.Holzl, A.Kharicha et al.// The $3^{\text {rd }}$ International Conference on Advances in Solidification Process, 2012. - p. 1-6.

[4] Frolov K.V. (Chairman et al). (1996) Mashinostroenie. Enciklopediy. Izmereniy, control, ispitaniy i diagnostika [Engineering Encyclopedia. Measurements, control, testing and diagnostics]: M, Engineering. -T.III,7 - 464 p.

[5] Ohser J. 3D-Images of Material Structures. Processing and Analysis/ Jaachim Ohser, Katja Schladitz. - 
Weinheim: WILEY-VCH Verlag Gmbh@Co K GaA, $2009-343 \mathrm{p}$

[6] Panteleyeva V.G., Yegorova O.V., Klikova Ye. (2005) Kompuyternay mikroskopiy [Computer Microscopy] M., Technosphera, -304 p.

[7] Prusov Ye.S. (2015) Komputernay tomografiy dly zadach trehmernogo materialovedeniy [Computer Tomography for Tasks of 3D Materials Sciense]. Fundamental Research - no 5, pp.318-323.

[8] Saai,A. Influence of volume fraction and distribution of martensite phase on the strain localization in dual phase steels/ A.Saaia, O.S.Hopperstad, Y.Granbom,O.-G Lademo// Procedia Materials Science, 2014. №3 p.900-905.

[9] Saltykov, S.A. (1970) Stereometricheskay metallografiy [Stereometrical metallography] - M: Metallurgy, - 376 p.

[10] Shteinberg S.S. (1961) Metallovedenie [Metal Science]. Sverdlovsk: Metallurgizdat, $1961-598$ p.

[11] Velichko,A. 3D characterization of Graphite Morphologies in Cast Iron/ Alexandra Velichko, Christian Holzapfel, Frank Muklich// Advanced Engineering Materials, 2007-9, №1-2 - p.39-45.

[12] Wu Q. Microscope Image Processing/ Qiang Wu, Fatima A.Merchant, Kenneth R. Castleman. Burlington: Academic Press is an Imprint of Elsevier, $2008-583$ p. 